\title{
Performance evaluation of graphite and titanium oxide powder mixed dielectric for electric discharge machining of Ti-6Al-4V
}

\author{
Shahriar Tanvir Alam ${ }^{1}$ (D) . AKM Nurul Amin ${ }^{1} \cdot$ Md. Ibrahim Hossain ${ }^{1} \cdot$ Maliha Huq $^{1} \cdot$ Shaqauit Hossain Tamim $^{1}$
}

Received: 12 November 2020 / Accepted: 25 February 2021 / Published online: 6 March 2021

(C) The Author(s) 2021 OPEN

\begin{abstract}
$\mathrm{Ti}-6 \mathrm{Al}-4 \mathrm{~V}$ is the most commonly used titanium alloy in aerospace, marine, and biomedical applications. Due to the properties of poor machinability in conventional machining, Electrical Discharge Machining (EDM) is considered a prospective alternative for machining this strategic material. This study aims at enhancing the performance of powder mixed EDM (PMEDM) in the machining of Ti-6Al-4V with the application of two different types of powders, namely Graphite (Gr) and Titanium Oxide $\left(\mathrm{TiO}_{2}\right)$ powders, with different concentrations in dielectric-kerosene. The effect of these powers and their relative quantities are studied in terms of metal removal rate (MRR), tool wear rate, Surface Roughness, and surface integrity. Machining is performed using the copper electrode and kerosene as the dielectric medium. A separate container and a submersible pump are used to limit the quantity of powder and keep the powder in suspension, respectively. Design of experiments guided by Design-Expert software is employed to minimize the number of experimental runs and develop empirical models of response parameters in terms of the variable parameters-peak current, powder type, and powder concentration. Findings indicate that $\mathrm{TiO}_{2}$ powder has a much higher effect on MRR compared to graphite powder, as the maximum MRR in the case of $\mathrm{TiO}_{2}$ powder is recorded $41.01 \mathrm{~mm} / \mathrm{min}$ against $11.98 \mathrm{~mm}^{3} / \mathrm{min}$ for graphite powder, i.e., 3.42 times higher. Similarly, the tool wear ratio for $\mathrm{TiO}_{2}$ powder is 0.0704 against 0.1219 for graphite powder at the maximum MRR, which is 1.73 times lower compared to that of graphite powder. The same ratios at the minimum MRR for $\mathrm{TiO}_{2}$ is 0.0098 , and for graphite power is 0.0282 , which is again 2.88 times lower compared to that of graphite powder. In terms of average surface roughness, $\mathrm{Ra}$, the performance of $\mathrm{TiO}_{2}$ is far better compared to graphite powder since the maximum surface roughness attained with $\mathrm{TiO}_{2}$ powder is $3.265 \mu \mathrm{m}$ against $9.936 \mu \mathrm{m}$ for graphite powder at the highest MRR and the same attained at the lowest MRR are $2.228 \mu \mathrm{m}$ and $2.411 \mu \mathrm{m}$ for TiO2 and graphite powders respectively. The mechanism of the effects of PMEDM on surface texture has also been observed using SEM images to study the influence of powder concentration on surface morphology.
\end{abstract}

Keywords Ti-6Al-4V $\cdot$ MRR $\cdot \mathrm{RSM} \cdot \mathrm{TWR} \cdot \mathrm{HAZ} \cdot \mathrm{SR}$

\section{Introduction}

Globalization has compelled manufacturers to invest more in developing sophisticated and quality products to meet the customer's ever-changing needs. Research in manufacturing has primarily focused on high-speed machining of complex parts involving CNC machines, chemical machining, laser beam machining (LBM), electron beam machining (EBM), and electron discharge machining (EDM), to keep pace with these needs [1]. The environment of chemical machining is hazardous, whereas LBM and EBM require considerable investments compared to EDM. EDM is the most preferred machining process for cutting extremely hard materials [2]. EDM is based on the

Shahriar Tanvir Alam, tanvir.shahriar.tro@gmail.com | ${ }^{1}$ Department of Industrial and Production Engineering, Military Institute of Science and Technology (MIST), Dhaka 1216, Bangladesh. 
electro-thermal principle for cutting electrically conducted hard materials [3]. During EDM machining, the work-piece and the tool are immersed in a dielectric fluid and are separated by a tiny gap, known as the spark gap [4]. When a voltage difference between the anode (the tool) and the cathode (the work-piece) is sufficient to break the resistance of the dielectric, an electrical discharge takes place [5]. The spark's energy generates high temperatures, up to $30,000^{\circ} \mathrm{C}$ on the work-pieces surface. This temperature causes the melting of a tiny part of the work-piece and vaporizing it [6]. There is no residual stress exist in the workpiece after an experimental run as there has not any contact between the tool and electrode [7]. Reasonable levels of finish can be achieved by employing very low pulse currents.

A relatively new advancement is the addition of powder in the dielectric fluid of EDM, which also improves MRR and lowers TWR significantly [8]. Powder mixed electrical discharge machining (PMEDM) has arisen as an efficient method in upgrading the abilities of EDM [9]. A combination of powder in EDM dielectric has become a feasible approach to overcome the significant EDM limitations and to produce a more refined surface finish over large process areas [10]. A mixture of suitable fine powder into the dielectric eases the dielectric's disintegration and, as a result, decreases its resistivity [2]. Furthermore, it facilitates more spontaneous sparks and eliminates abnormal discharge by producing a much better surface finish. Various researchers tested powder of different materials to check the performance of PMEDM [11]. Findings identify that the PMEDM may distinctly increase the surface quality and provide a mirror-like surface finish at a comparatively high machining rate [12].

Titanium alloy has high specific strength, good process performance, corrosion resistance, and the ability to retain its mechanical properties at high temperatures which is the perfect material for aerospace engineering [13]. However, Ti-6Al-4V is difficult-to-cut material with poor machinability using traditional machining approaches, while EDM is best suited for machining Ti-6AI-4V [14]. The tool must be 3-4 times harder than the work-piece, and it has to have diffusion and fatigue resistance, which is very difficult to maintain in the case of machining of Ti-6AI-4V with conventional machining process [15]. Physical contact of the tool is not needed; consequently, cutting forces are not involved; instead, material removal is done through electrical energy in EDM. Hwang et al. [16] discussed the response of utilizing multi-layered electrodes with turn reverse polarity in electric discharge measure. The layer of TiC layer on the outside of the workpiece (nickel) brings about expanded surface hardness, along with diminished microcracks [17]. Khan et al. [18] analyzed the surface integrity of the PMEDM process with two different powders ( $\mathrm{TiC}$ and $\mathrm{Al}_{2} \mathrm{O}_{3}$ ). Janmanee and Muttamara [19] investigated the coating of Ti on tungsten-carbide surface applying PMEDM process where Ti powder is used as the dielectric. So, it raises the importance of investigating the performance of non-conventional machining such as PMEDM of Ti-6Al-4V using Graphite and $\mathrm{TiO}_{2}$ Powder.

The main objective of this study is to investigate the effects of pulse energy (current), powder concentration, and powder type on MRR, SR, and TWR in the PMEDM process of Ti-6Al-4V. In this work, fine powders of graphite and $\mathrm{TiO}_{2}$ are mixed with kerosene (dielectric) in different quantities. Response surface methodology (RSM) is conducted to generate the experimental runs. Three crucial performance measures, i.e., MRR, TWR, and SR, are considered to assess process parameters' influence. Finally, a detailed investigation on the surface topography is carried out using scanning electron microscope (SEM) to understand the morphology of the HAZ under different PMEDM conditions.

The research paper is organized as: Sect. 2 presents the materials and methods of this work. Section 3 shows the results and discusses the findings of this study, and Sect. 4 represents the conclusion and recommendation for future works.

\section{Materials and methods}

\subsection{Work material}

Grade $5 \mathrm{Ti}-6 \mathrm{Al}-4 \mathrm{~V}$ bars are selected as work-piece material. The chemical composition of Ti-6Al-4V is shown in Table 1 [20]. The physical, mechanical, thermal \& electrical properties of the material are exhibited in Table 2 [21].

\subsection{The powder used in the PMEDM process}

The electrically conductive powder is mixed with a dielectric of EDM, reducing the insulating strength of dielectric fluid

Table 1 Ti-6A1-4V (Grade 5) Material Composition [20]

\begin{tabular}{ll}
\hline Component & Composition (wt\%) \\
\hline Titanium & $86-90$ \\
Aluminum & $5.5-6.75$ \\
Vanadium & $3.5-4.5$ \\
Iron & Max 0.40 \\
Oxygen & Max 0.20 \\
Carbon & Max 0.80 \\
Nitrogen & Max 0.05 \\
Hydrogen & Max 0.0125 \\
Other elements (Total) & 0.40 \\
\hline
\end{tabular}


Table 2 Properties of Titanium Ti-6Al-4V (Grade 5) [21]

\begin{tabular}{ll}
\hline Properties & Metric \\
\hline Density & $4.43 \mathrm{~g} / \mathrm{cc}$ \\
Atomic volume & $0.01 \mathrm{m3} / \mathrm{kmol}$ \\
Hardness, brinell & 334 \\
Tensile strength, ultimate & $1000 \mathrm{MPa}$ \\
Tensile strength, yield & $895 \mathrm{MPa}$ \\
Elongation at break & $9 \%$ \\
Modulus of elasticity & $113.8 \mathrm{GPa}$ \\
Poisson's ratio & 0.342 \\
Specific heat capacity & $0.5263 \mathrm{~J} / \mathrm{g}-\mathrm{C}$ \\
Thermal conductivity & $6.7 \mathrm{~W} / \mathrm{m}-\mathrm{K}$ \\
Electrical resistivity & $0.0001782-\mathrm{cm}$ \\
Magnetic permeability & 1.00005 \\
Magnetic susceptibility & $3.3 \mathrm{e}-006$ \\
\hline
\end{tabular}

and increasing the spark gap between the tool and workpiece in the PMEDM process [22, 23]. Micro-sized fine powders of graphite $(\mathrm{Gr})$ and Titanium oxide $\left(\mathrm{TiO}_{2}\right)$ are selected for the experiment. The solubility of $\mathrm{Gr}_{\text {and }} \mathrm{TiO}_{2}$ in kerosene is not the same, for which initiation of electric spark and discharge varies. Hence, variation in responses like MRR and TWR occurs.

\subsection{Experimental setup}

A submersible 12V 8-W DC centrifugal brush-less Solar pump with hermetically sealed motor close-coupled with the pump body was used inside a small box containing a limited dielectric quantity-kerosene. The pump's primary purpose is to maintain a constant flow of the dielectric in the machining zone and remove the debris before depositing it on the material's surface during machining, thereby ensuring spark efficiency. The experiment was conducted on an Electric Discharge Machine-JS EDM NCF606N. DOE was applied for the study, a set of approaches that revolve around influencing factors on the experiment's response. Input factors selected in this work were Peak Current, the powder used, and powder concentration. The value was taken for "without powder" was 0 , whereas 1 and 2 for $\mathrm{TiO} 2$ powder and graphite powder, respectively. The peak current used during all the tests ranges from 4 to 20A. The choice of the peak current interval was made, considering the
Table 4 Output factors of the experiments

\begin{tabular}{ll}
\hline Name & Units \\
\hline MRR & $\mathrm{mm}^{3} / \mathrm{min}$ \\
TWR & $\mathrm{mm}^{3} / \mathrm{min}$ \\
SR & micro m \\
\hline
\end{tabular}

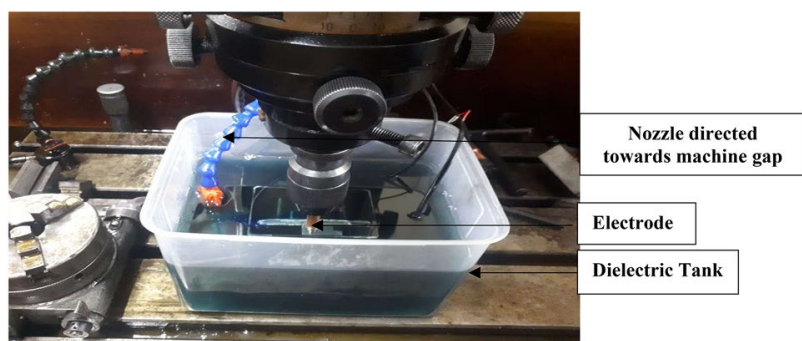

Fig. 1 Experimental Setup of the PMEDM

PMEDM process characteristics and work-piece materials. Along with this, the cutting conditions in Table 3 were selected based on contemporary works. Moreover, for selecting the ranges of cutting conditions, few trial runs were conducted. Table 4 represents the output factors of the experiments. Kansal et al. [23] mentioned that 'Despite the promising results, PMEDM process is used in industry at a very slow pace.' Among the many recommendations made by the authors is the need for optimization of the concentration of the working fluid. The current paper intended to address this issue and also to identify a suitable power for application in PMEDM. Apart from that, the empirical equations developed in the work will help in predicting the MRR, TWR, SR at a 95\% confidence level. The developed approach will help in deriving a similar relationship for the PMEDM responses as functions of its other variable parameters. Figure 1 illustrates the experimental setup. Pulse-on/off time is a crucial factor affecting the output responses of the EDM, which were set at a constant value of $55 \mu \mathrm{s}$; however, due to the defined scope of the present work, it was decided to maintain it at a default value. The pulse on/off span was chosen based on control board settings accessible on the machine and literature review [24].

The following process diagram (Fig. 2) was applied to find the optimum machining conditions in this study.
Table 3 Input factors of the experiments

\begin{tabular}{lllllll}
\hline Input parameter & Name & Units & Type & Levels & $\mathrm{L}(1)$ & $\mathrm{L}(2)$ \\
\hline A (Numeric) & Peak current & $(\mathrm{A})$ & Continuous & $\mathrm{N} / \mathrm{A}$ & 4 & 20 \\
B (Numeric) & Powder conc & $(\mathrm{g} / \mathrm{L})$ & Continuous & $\mathrm{N} / \mathrm{A}$ & 5 & 9 \\
C (Categorical) & Powder used & $(\mathrm{Ct})$ & Nominal & 2 & $\mathrm{Gr}$ & $\mathrm{TiO} 2$ \\
\hline
\end{tabular}




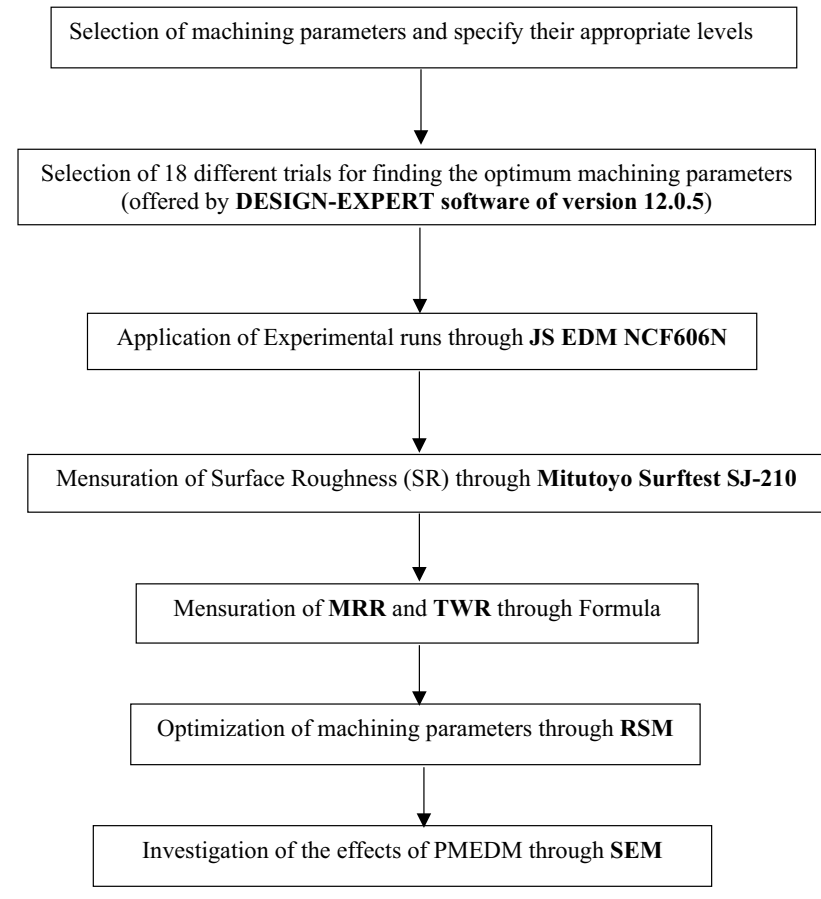

Fig. 2 A process diagram of this study

\section{Results and discussion}

\subsection{Response surface methodology}

Response surface methodology (RSM) is an approach that can be conducted to generate an optimized process with a collection of statistical and mathematical processes. RSM helps express empirical relations of cutting parameters and their interaction on the considered responses [25]. The calculations for the RSM model development were carried out through the Design-Expert 12.0.5 software. Table 5 shows the experimental conditions and the measured response results. The regression calculation(s) to fit all polynomial models to the selected response (MRR, TWR, SR) was performed in the Fit and Summary test. It calculates the effects of all the model terms. One experimental "Run" was conducted once with a fixed combination of cutting conditions, which was developed by the DesignExpert 12.0.5 software.

Surface roughness (SR) was calculated using Mitutoyo Surftest SJ-210. The surface roughness of the machined surface was measured perpendicular to that feed after the individual experimental run. Three readings of surface roughness were averaged for each of the 18 different combinations of the experimental run.

Table 5 DOE table using Response Surface Methodology

\begin{tabular}{|c|c|c|c|c|c|c|c|}
\hline Std. Run & Run & $\begin{array}{l}\text { Fact } 1 \text { peak cur- } \\
\text { rent }(A)\end{array}$ & $\begin{array}{l}\text { Fact } 2 \text { powder con- } \\
\text { centration }(\mathrm{g} / \mathrm{L})\end{array}$ & $\begin{array}{l}\text { Fact3 powder } \\
\text { type (Ct.) }\end{array}$ & $\begin{array}{l}\text { Response1 MRR } \\
(\mathrm{mm} 3 / \mathrm{min})\end{array}$ & $\begin{array}{l}\text { Response2 TWR } \\
(\mathrm{mm} 3 / \mathrm{min})\end{array}$ & $\begin{array}{l}\text { Response3 } \\
\text { SR (micro m) }\end{array}$ \\
\hline 15 & 1 & 20 & 9 & $\mathrm{TiO} 2$ & 38.221 & 0.229 & 2.681 \\
\hline 3 & 2 & 12 & 9 & $\mathrm{Gr}$ & 7.921 & 0.089 & 6.246 \\
\hline 7 & 3 & 20 & 5 & $\mathrm{Gr}$ & 11.98 & 0.146 & 3.511 \\
\hline 5 & 4 & 4 & 7 & $\mathrm{TiO} 2$ & 10.593 & 0.088 & 2.631 \\
\hline 18 & 5 & 20 & 7 & $\mathrm{Gr}$ & 6.885 & 0.185 & 9.936 \\
\hline 16 & 6 & 12 & 7 & $\mathrm{Gr}$ & 6.215 & 0.001 & 4.583 \\
\hline 4 & 7 & 12 & 7 & $\mathrm{TiO} 2$ & 25.265 & 0.039 & 2.554 \\
\hline 10 & 8 & 20 & 9 & $\mathrm{Gr}$ & 11.263 & 0.217 & 5.625 \\
\hline 17 & 9 & 20 & 7 & $\mathrm{TiO} 2$ & 41.012 & 0.289 & 3.265 \\
\hline 14 & 10 & 4 & 5 & Gr & 2.411 & 0.068 & 2.541 \\
\hline 1 & 11 & 4 & 9 & $\mathrm{Gr}$ & 4.564 & 0.006 & 3.238 \\
\hline 2 & 12 & 12 & 5 & $\mathrm{TiO} 2$ & 14.935 & 0.026 & 2.584 \\
\hline 6 & 13 & 4 & 7 & $\mathrm{Gr}$ & 4.213 & 0.004 & 3.56 \\
\hline 9 & 14 & 4 & 9 & $\mathrm{TiO} 2$ & 12.235 & 0.083 & 2.228 \\
\hline 8 & 15 & 20 & 5 & $\mathrm{TiO} 2$ & 21.246 & 0.336 & 3.225 \\
\hline 12 & 16 & 4 & 5 & $\mathrm{TiO} 2$ & 9.214 & 0.091 & 3.224 \\
\hline 13 & 17 & 12 & 9 & $\mathrm{TiO} 2$ & 36.012 & 0.193 & 2.589 \\
\hline 11 & 18 & 12 & 5 & $\mathrm{Gr}$ & 7.625 & 0.157 & 3.985 \\
\hline
\end{tabular}


MRR was calculated by measuring each workpiece before the experimental run and after each operation on EDM, when the part with the dielectric liquid was cleaned. Equation 1 was used with a sensitive device (Denver Instrument) having an accuracy of ( $\pm 0.001 \mathrm{gm}$ ) for all the MRR $\left(\mathrm{mm}^{3} / \mathrm{min}\right)$ values $[26,27]$.

$\operatorname{MRR}=\frac{W_{i}-W_{f}}{\rho_{w} t}$

where, $W_{i}(\mathrm{gm})$ represents the initial weight, and $W_{f}(\mathrm{gm})$ shows the terminal weight of the experimental workpiece. $\rho_{w}\left(\mathrm{gm} / \mathrm{mm}^{3}\right)$ represents the density of the workpiece $(4.43 \mathrm{~g} / \mathrm{cc})$, and $t(\mathrm{~min})$ shows the time interval of the experimental run.

TWR was calculated by a similar approach with the electrode. Equation 2 was used for all the TWR $\left(\mathrm{mm}^{3} / \mathrm{min}\right)$ values [28].

$\operatorname{TWR}=\frac{W_{i e}-W_{f e}}{\rho_{e} t}$

where $W_{i e}(\mathrm{gm})$ represents the initial weight, and $W_{f e}(\mathrm{gm})$ shows the terminal weight of the electrode. $\rho_{e}\left(\mathrm{gm} / \mathrm{mm}^{3}\right)$ represents the density of the electrode.

\subsection{Analysis of variance (ANOVA) analysis}

The analysis of variance (ANOVA) was conducted to check the formulated model's ampleness developed by using the Design-Expert 15.0.5 software, which provides statistics such as $p$-values and F-values for comparing the models. Table 6 illustrates the ANOVA table for main and interaction effects on MRR during the experimental study.

The Model F-value of 14.29 implies that the model is significant. There is only a $0.03 \%$ chance that a "Model F-value" this large could occur due to noise. $P$-values of less than 0.0500 indicate that those model terms are significant, which means $A, B, A B, A^{2}$, and $B^{2}$ are significant terms. ANOVA analysis was conducted for the other response (TWR), and the findings were the Model F-value for TWR is 3.64, which implies the model is significant and $A, B, A B, A^{2}$, and $B^{2}$ are significant model terms in formulating the TWR equation as their $P$-values are less than 0.0500 . Table 7 represents the comprehensive statistics of the experimental values and design summary.

Six equations were generated for two categories of factors. These equations in terms of the fundamental factors for $\mathrm{Gr}$ and $\mathrm{TiO}_{2}$ suspended dielectric is shown in Eqs. (3)-(8). These equations can be used to make predictions about the responses for given levels of each factor. According to the fit and summary tests, quadratic models were suggested for Eq. (3)-(6), while linear models are suggested for Eqs. (7), (8), where A represents the peak current $\left(\mathrm{I}_{0}\right)$, and $\mathrm{B}$ represents the powder concentration. The equations are shown below:

$$
\begin{aligned}
(\mathrm{MRR})_{\mathrm{Gr}}= & -4.74618+0.479333 \times \mathrm{A} \\
& +2.23742 \times \mathrm{B}+0.086594 \times \mathrm{A} * \mathrm{~B} \\
& -0.028788 \times \mathrm{A}^{2}-0.223729 \times \mathrm{B}^{2}
\end{aligned}
$$

Table 6 ANOVA for main and interaction effects on MRR during EDM of Ti-6Al-4 V

\begin{tabular}{lcccccc}
\hline Source & Sum of squares & Df & Mean square & F-value & $p$-value (Prob > F) & Remark \\
\hline Model & 2332.84 & 8 & 291.61 & 14.29 & 0.0003 & $\begin{array}{c}\text { Signifi- } \\
\text { cant }\end{array}$ \\
A-peak current & 636.23 & 1 & 636.23 & 31.18 & 0.0003 & \\
B-powder concentration & 152.69 & 1 & 152.69 & 7.48 & 0.0230 & \\
C-powder used & 1178.65 & 1 & 1178.65 & 57.77 & 0.0001 & \\
AB & 15.36 & 1 & 15.36 & 0.7527 & 0.0408 \\
AC & 13.58 & 1 & 13.58 & 0.6655 & 0.4357 \\
BC & 3.20 & 1 & 3.20 & 0.1570 & 0.7011 \\
A & 204.16 & 1 & 204.16 & 10.01 & 0.0115 \\
B & 128.98 & 1 & 128.98 & 6.32 & 0.0331 & \\
Residual & 183.62 & 9 & 20.40 & & & \\
Cor Total & 2516.46 & 17 & & & &
\end{tabular}

Table 7 Design summary of the responses

\begin{tabular}{lllccll}
\hline Name & Observations & Minimum & Maximum & Std. dev & Transform & Model \\
\hline MRR & 18 & 2.411 & 41.012 & 12.17 & Power & Quadratic \\
TWR & 18 & 0.001 & 0.336 & 0.1002 & None & Quadratic \\
SR & 18 & 2.228 & 9.936 & 1.88 & None & Linear \\
\hline
\end{tabular}




$$
\begin{aligned}
(\mathrm{MRR})_{\mathrm{TiO} 2}= & -23.88535+1.51052 \times \mathrm{A} \\
& +5.51583 \times \mathrm{B}+0.086594 \times \mathrm{A} \times \mathrm{B} \\
& -0.028788 \times \mathrm{A}^{2}-0.223729 \times \mathrm{B}^{2}
\end{aligned}
$$

In these equations, no transformation was used for peak current and powder concentration; thus, the factor with the highest value of coefficient possesses the most dominating effect over the response of MRR.

$$
\begin{aligned}
(\mathrm{TWR})_{\mathrm{Gr}}= & 0.546917-0.014943 \times A-0.133229 \\
& \times \mathrm{B}+0.000266 \times A \times B+0.000953 \\
& \times A^{2}+0.008937 \times \mathrm{B}^{2}
\end{aligned}
$$

$(\mathrm{TWR})_{\mathrm{TiO} 2}=0.507333-0.012401 \times \mathrm{A}$

$$
\begin{aligned}
& -0.123979 \times B+0.000266 \times A \times B \\
& +0.000953 \times A^{2}+0.008937 \times B^{2}
\end{aligned}
$$

$(\mathrm{SR})_{\mathrm{Gr}}=2.41853+0.112719 \times \mathrm{A}+0.147375 \times \mathrm{B}$

$(\mathrm{SR})_{\mathrm{TiO} 2}=0.391417+0.112719 \times \mathrm{A}+0.147375 \times \mathrm{B}$

\subsection{Effect of process parameters on MRR, TWR, and SR}

A response surface plot is a tool providing an estimation of the optimum response region, which is similar to the $3 D$ response graph [29]. The 3D response graph of MRR (Fig. 3a) has been plotted with the data collected for the

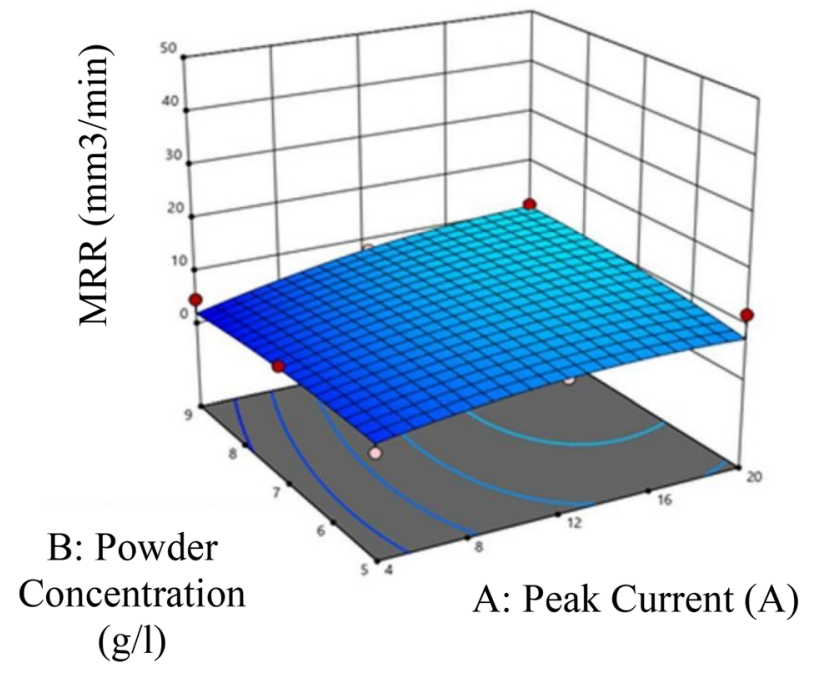

(a) Graphite

Fig. 3 Graph plotted for MRR of $\mathbf{a} \mathrm{Gr} \& \mathbf{b} \mathrm{TiO}_{2}$ powder
Gr powder, and (Fig. 3b) has been plotted with the data collected for $\mathrm{TiO}_{2}$ powder. It is observed that the MRR in the case of $\mathrm{TiO}_{2}$ powder is much higher (approximately 4 times) compared to that of Gr powder. It may be noted here that the 3D plots are an average approximation of the MRR values for the combination of the parameters for $\mathrm{Gr}$ and $\mathrm{TiO}_{2}$ powders. It may be noted here that minor local effects of the parameters have been averaged in these plots during the regression process.

Contour graphs, i.e., the projections of 3D graphs on a 2D plot, show the upper and lower points and the effect of two factors on a specific response. The graphs of Fig. 4 show how variable $A$ (peak current) and variable $B$ (powder concentration) affect the TWR in the case of Gr powder (a) and $\mathrm{TiO}_{2}$ powder (b). The blue region in the $3 \mathrm{D}$ graph indicates the lowest TWR, the green, and yellow region indicates moderate values, and the red region indicates the highest TWR. It is observed from the plots of Fig. 4 that the TWR is slightly lower (1.5 times) when Gr powders are used compared to that when $\mathrm{TiO}_{2}$ is used. However, comparing the much higher metal removal rate (MRR) (4.5 times) in $\mathrm{TiO}_{2}$ powder, its slightly more significant influence on TWR may be ignored.

3D response graph of average surface roughness (SR) for the Gr powder graph is shown in Fig. 5a and that for $\mathrm{TiO}_{2}$ powder in Fig. $5 \mathrm{~b}$. The variables of the contour graphs are peak current $(A)$ and powder concentration (B). The two plots show that the surface roughness is lower for $\mathrm{TiO}_{2}$ powder compared to $\mathrm{Gr}$ powder. In both, cases peak current has a more significant influence on SR compared to powder concentration. It may be further noted that

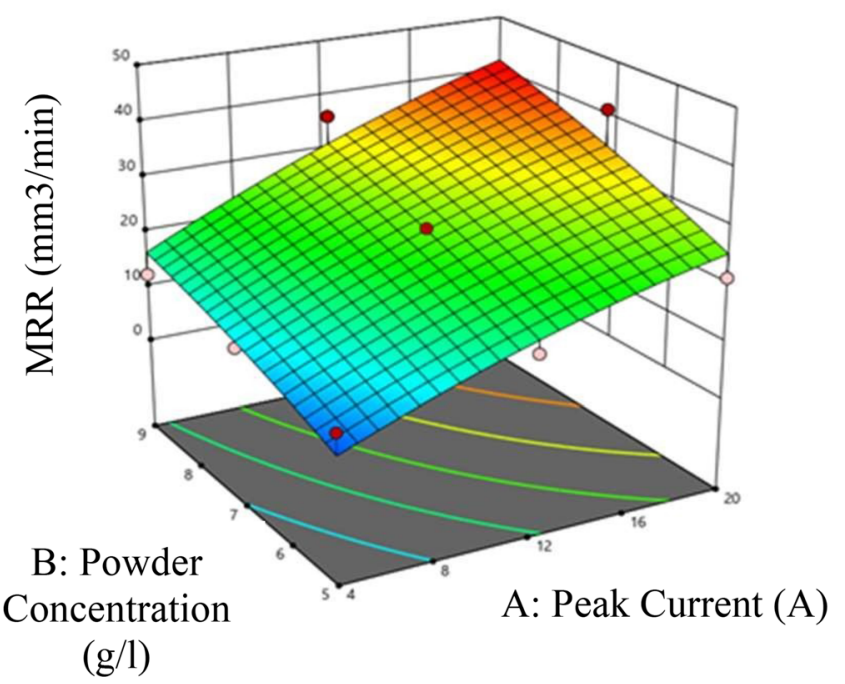

(b) Titanium Oxide 

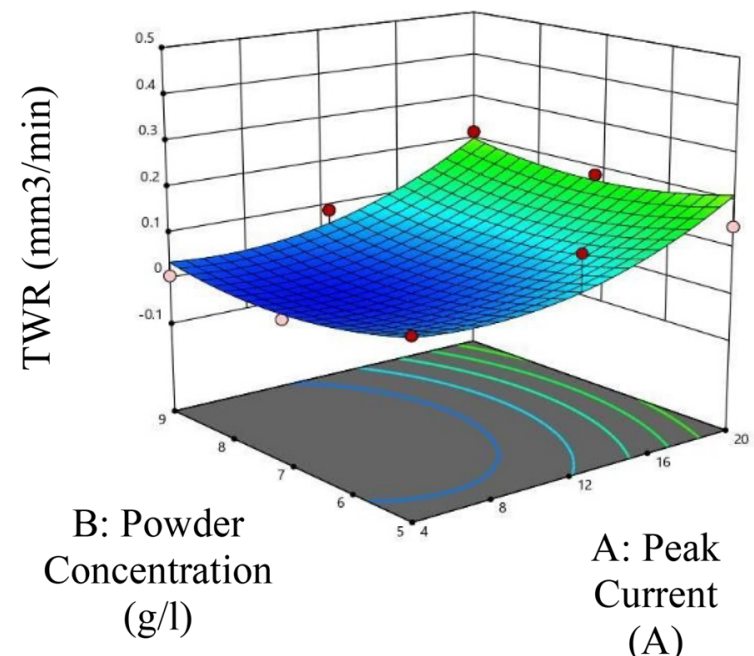

(a) Graphite

Fig. 4 Graph plotted for TWR for $\mathbf{a} \mathrm{Gr}, \mathbf{b} \mathrm{TiO}_{2}$ powder

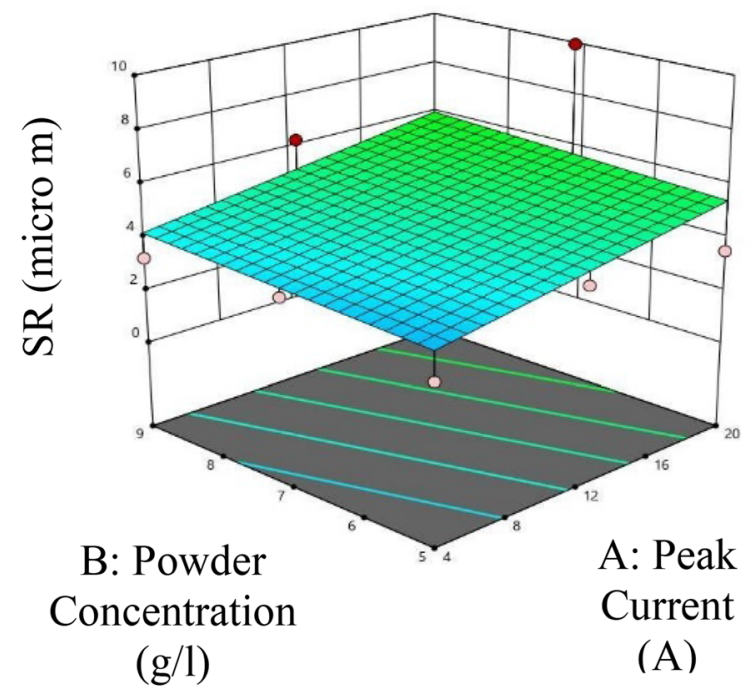

(a) Graphite

Fig. 5 Graph plotted for SR of $\mathbf{a} \mathrm{Gr} \& \mathbf{b} \mathrm{TiO}_{2}$ powder

though the MRR in the case of $\mathrm{TiO}_{2}$ powders is approximately 3.5 times higher than that of Gr powder, that did not lead to higher surface roughness generation; on the contrary, lower surface roughness (approximately 1.5 times lower) is achieved in the case of $\mathrm{TiO}_{2}$ powder. Again, it may be noted that minor local effects of the parameters have been averaged out in the 3D plots during the regression process.

As there is a three-hold limit of powder concentration, depending on each powder's properties, some of the

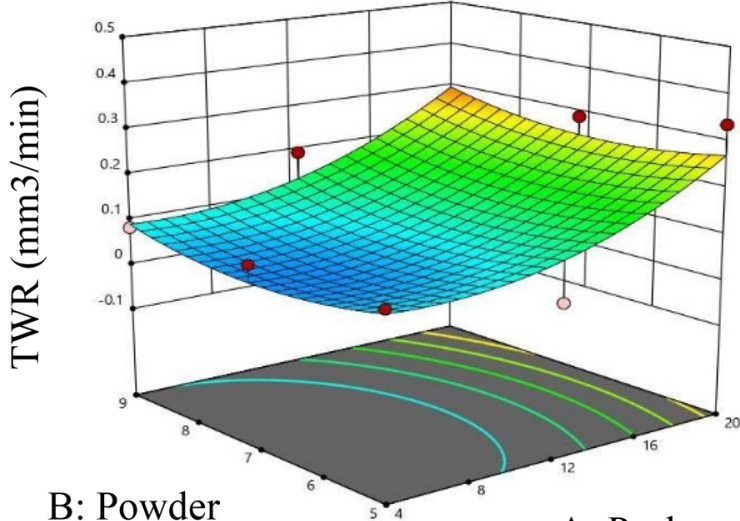

A: Peak

Current

(A)

(b) Titanium Oxide

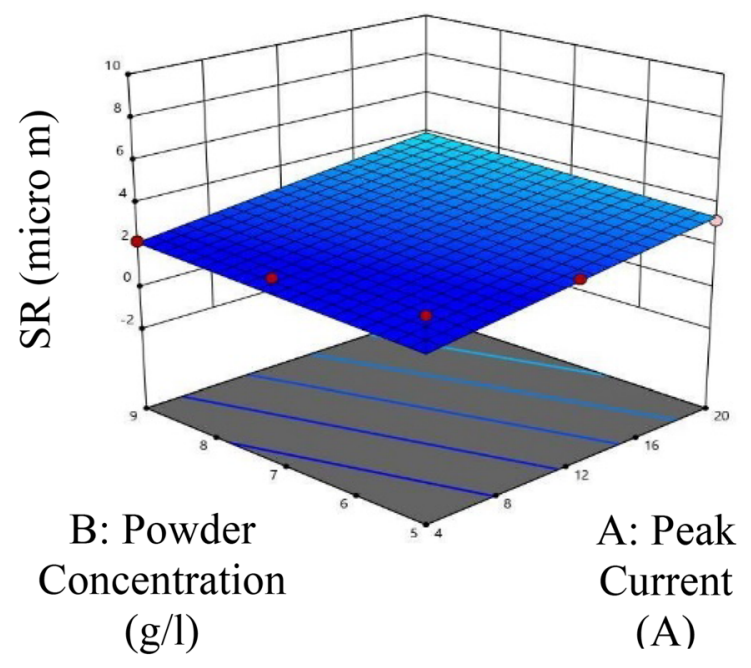

(b) Titanium Oxide

values of roughness are more in the additive machining process than the normal process. In such cases, the powder particles were heavily in between the spark gap, which decreases the smoothness of the surface created. It is visible that when the peak current is increased roughness value also increases. This is because when the peak current is raised, discharge energy per pulse is increased. As a result, a rough surface is produced at higher discharge energy. 


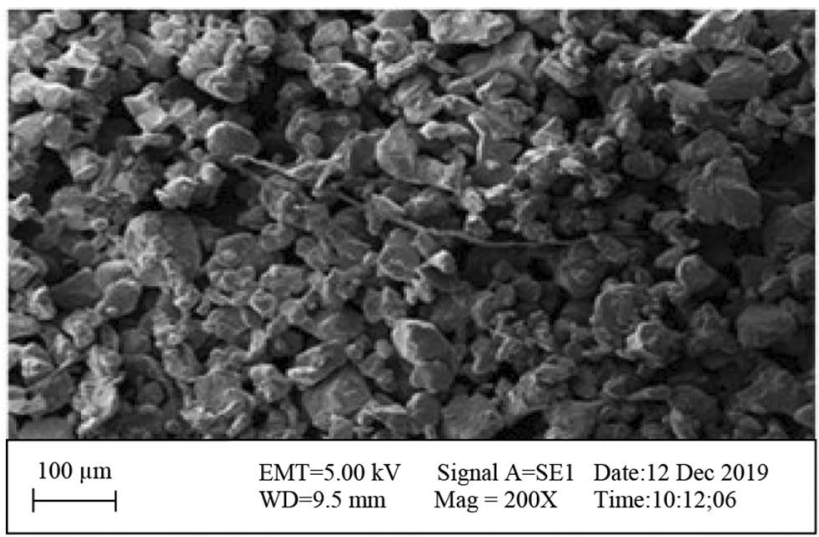

(a)

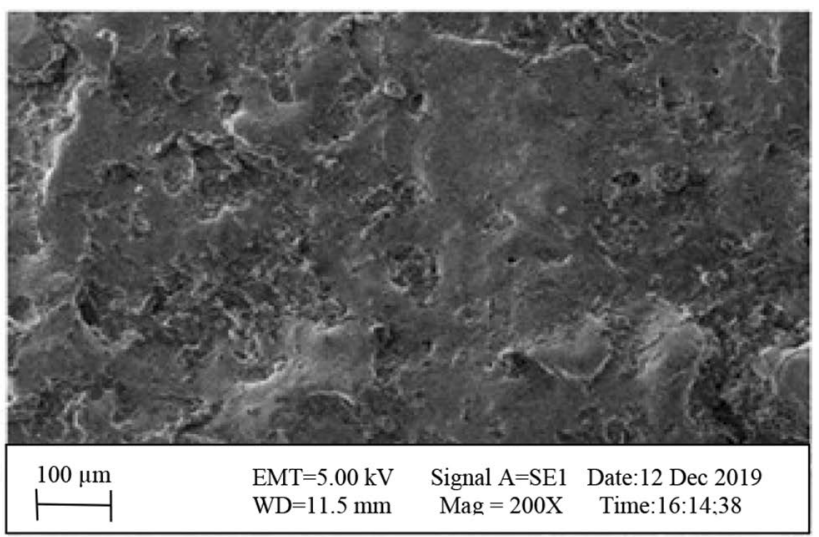

(b)

Fig. 6 Comparison of SR for $\mathbf{a}$ kerosene and $\mathbf{b}$ powder suspended kerosene used as the dielectric for different peak current $7 \mathrm{gm} / \mathrm{L}$ concentration of graphite at $\mathbf{a} \mathrm{Ip}=4 \mathrm{~A}$

\subsection{Investigation of the effects of PMEDM parameters on machined surface topography using SEM}

It is verified that using SEM, every detail of the chip can be viewed using very high magnification [30]. To investigate the effects of powder type, powder concentration, and peak current on the machined surface, views of the surface were observed using scanning electron microscope (SEM). The first set of experiments' main objectives were to understand the effect of powder on EDM performance. SEM images of Fig. $6 a$, b compare the surface topography under plain EDM using kerosene as the dielectric with the topography produced under PMEDM with $7 \mathrm{gm} / \mathrm{L}$ concentration of graphite powder a peak current of $4 \mathrm{~A}$. It is observed that there is a visible difference in the topography of the surfaces produced under the two conditions. A matted surface is shown in Fig. $6 \mathrm{~b}$ indicates that a smoother surface generated in PMEDM compared to the deep-rooted grooves of individual sparks (Fig. 6a). Material Removal Rate under these two conditions are almost the same as powder concentration does not appreciably influence the MRR, as shown in Fig. 3a. However, powder concentration tends to decrease surface roughness. In PMEDM, the powder particles enter into the spark gap and create a bridging effect. As a result, the plasma channel becomes enlarged and widened. The material removed takes place smoothly over a larger surface area. Thus, powder particles' presence results in a lower depth of the crater, taking place over a wider area, and consequently, the polishing time and the surface roughness are reduced [31]. Since the density of $\mathrm{TiO}_{2}$ powder is $4.23 \mathrm{~g} /$ $\mathrm{cm}^{3}$ and that of graphite powder is $1.8 \mathrm{~g} / \mathrm{cm}^{3}$, the volume percent of graphite is 2.35 times higher compared to the
$\mathrm{TiO}_{2}$ powder for the same weight percentage. Therefore, a larger number of graphite powder will be in the spark gap to influence the sparking process. However, graphite is a good conductor of electricity. When the voltage difference is set up in the spark gap, positively charged graphite powder particles will have the tendency to be attracted towards the cathode (electrode); hence the formation of a 'chain-like structure' to help in overcoming the dielectric strength of the medium (kerosene) may not be easily facilitated. However, in the case of $\mathrm{TiO}_{2}$ powder, which is a semi-conductive material, the induced bonding is more readily developed to form the 'chain-like structure' to overcome the dielectric strength of the medium. This may be the principal reason for higher MRR in the case of $\mathrm{TiO}_{2}$ powder, though the volume percent of the powder is 2.35 times lower compared to that of graphite powder at the constant weight percentage of the two powders. However, the phenomenon needs a more detailed study to arrive at a definite conclusion on the mechanism of the process.

\subsection{Investigation of the effect of peak current on HAZ in PMEDM at the powder concentration of $7 \mathrm{gm} / \mathrm{L}$}

To study the effect of peak current on surface topography and the expected HAZ, SEM images of the machined surface were taken (Fig. 7) for surfaces generated under different values of peak current $(4 \mathrm{~A}, 12 \mathrm{~A}$, and $20 \mathrm{~A})$ at a constant concentration of Graphite powder $(7 \mathrm{gm} / \mathrm{L})$. It is revealed that large areas of relatively smooth surfaces are generated at low current values of 4A (Fig. 7a) and 12A (Fig. 7b). On the other hand, the rougher surface is generated when the current values of $20 \mathrm{~A}$ are applied for the same concentration of the power, $7 \mathrm{gm} / \mathrm{L}$ (Fig. 7c). At lower 


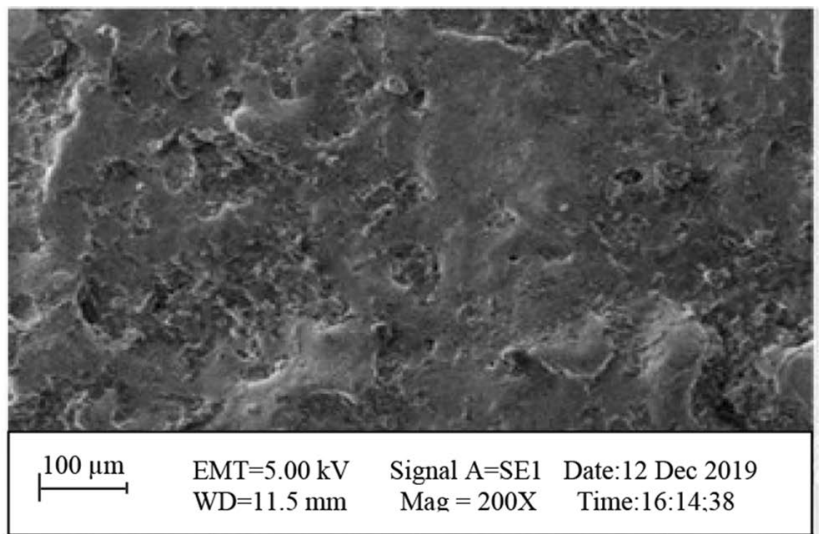

(a)

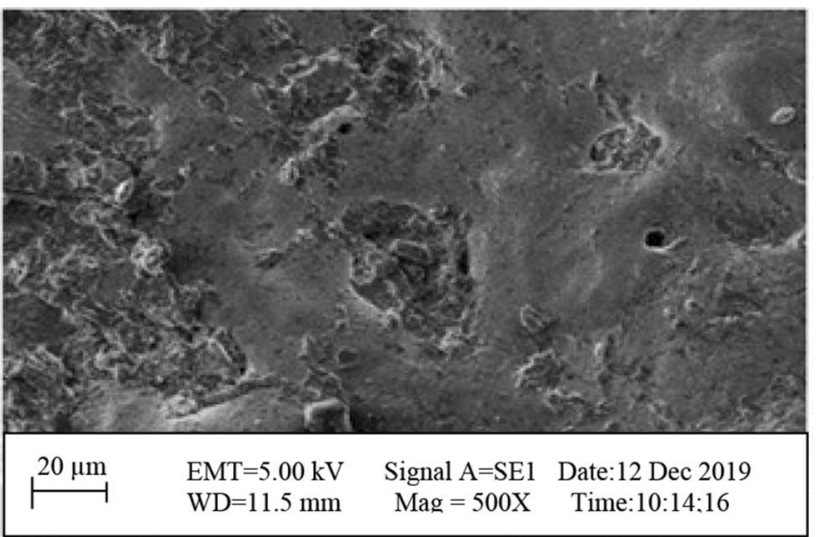

(b)

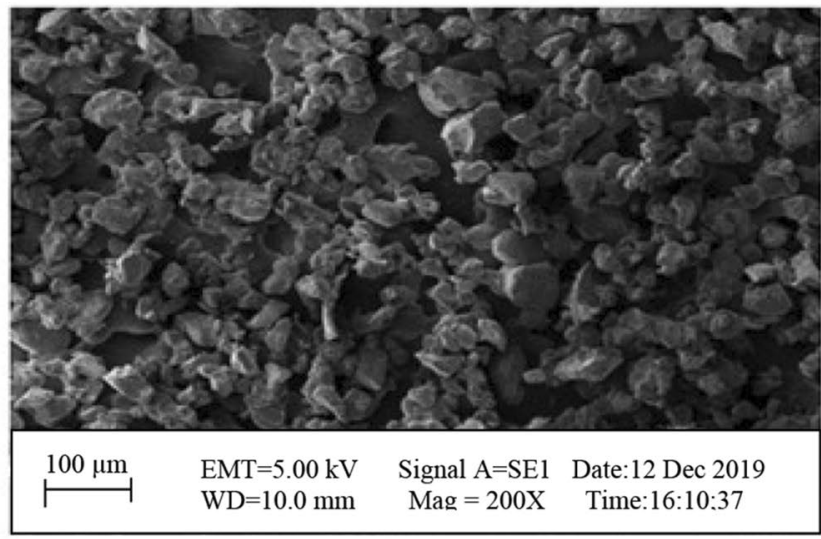

(c)

Fig. 7 SEM images for $7 \mathrm{gm} / \mathrm{L}$ concentration of graphite at $\mathbf{a}|\mathrm{p}=4 \mathrm{~A}, \mathbf{b}|=12 \mathrm{~A}$ and $\mathbf{c} \mid \mathrm{p}=20 \mathrm{~A}$

peak current values, shallower craters are created at low discharge energy compared to higher discharge energy. Comparing these images with those of Fig. $6 a$, b, it may be concluded here that the application of high peak current even in PMEDM leads to larger crater sizes, which results in higher surface roughness and greater depth of the HAZ in PMEDM. Hence the peak current value must be limited to a finite value to get the desired effect of PMEDM in better surface finish and lower depth of the HAZ.

\subsection{Investigation of the effect of powder concentration on HAZ in PMEDM at the highest peak current of $20 \mathrm{~A}$}

"Detailed analysis of structural features of the machined surface is done by using SEM to realize the mode of the recast layer thickness, $\mathrm{HAZ}$, and micro-cracks, which alternatively influences the structure of machined workpiece and hence tool life" $[32,33]$. To identify the influence of the concentration of the Graphite powder on the topography of the machined surface and the HAZ at the highest peak current of 20A, SEM images of the machined surfaces produced under different powder concentrations are compared in Fig. $8 \mathrm{a}-\mathrm{c}$. It is observed from these three images that a higher powder concentration is required to get the desired effect of PMEDM when a high peak current is used. In the case of $20 \mathrm{~A}$ current, no effect of powder is observed on the surface topography when the concentrations are $5 \mathrm{gm} / \mathrm{L}$ (Fig. 8a) and $7 \mathrm{gm} / \mathrm{L}$ (Fig. 8b). However, the beneficial effect of PMEDM is observed on the surface topography when the powder concentration is increased to $9 \mathrm{gm} / \mathrm{L}$ (Fig. $8 \mathrm{c}$ ).

As a result of this, a sharp reduction of surface roughness (from 9.9 to $5.6 \mu \mathrm{m}$ ) is observed when the Graphite powder concentration increases from 7 to $9 \mathrm{gm} / \mathrm{L}$ which can be seen in the bar chart of Fig. 9 and Table 5. Similar effects are also observed in the case of $\mathrm{TiO}_{2}$ powder, as shown in the same figure and Table. However, this reduction in surface roughness is not caused at the cost of lower MRR. On the contrary, MRR is higher when power 


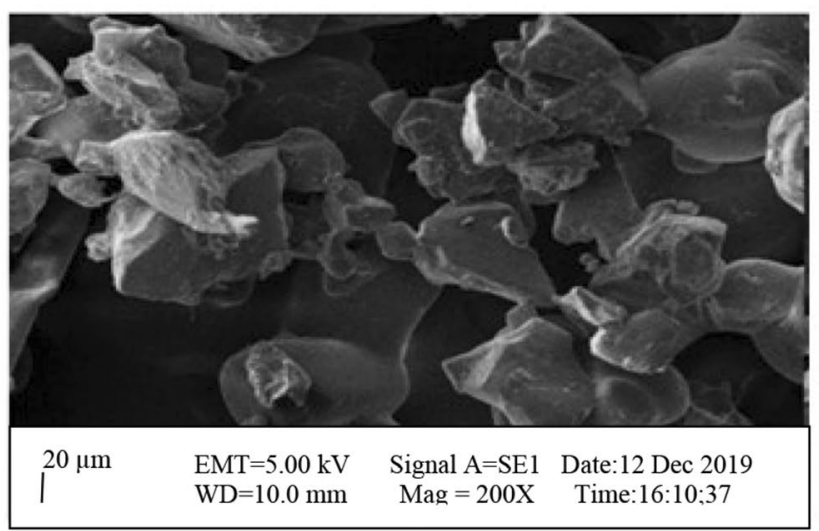

(a)

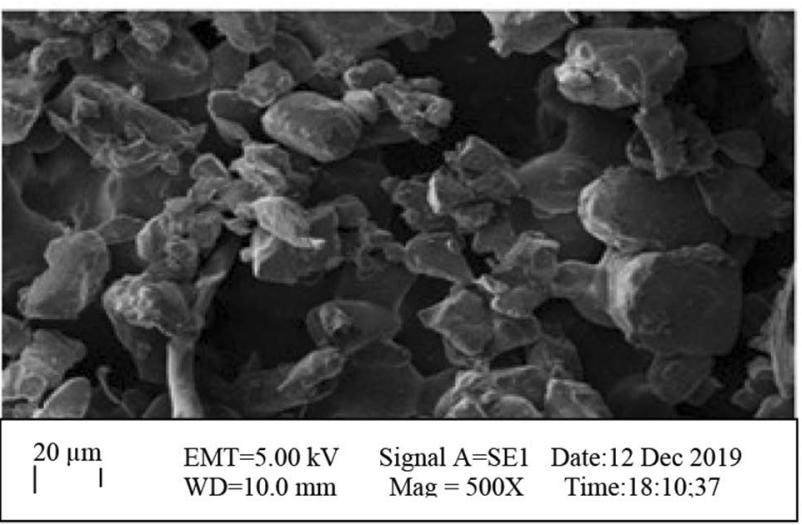

(b)

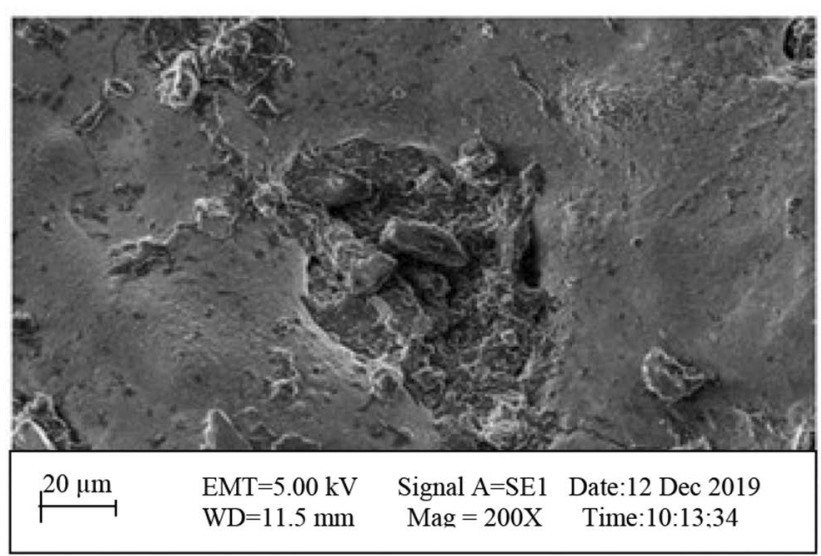

(c)

Fig. 8 SEM images for $\mathrm{Ip}=20 \mathrm{~A}$ for different concentrations a $5 \mathrm{gm} / \mathrm{L}, \mathbf{b} 7 \mathrm{gm} / \mathrm{L}$ and $\mathbf{c} 9 \mathrm{gm} / \mathrm{L}$ of graphite

Influence of Powder concentration on Surface Roughness (SR) for $\mathrm{Gr}$ and $\mathrm{TiO} 2$

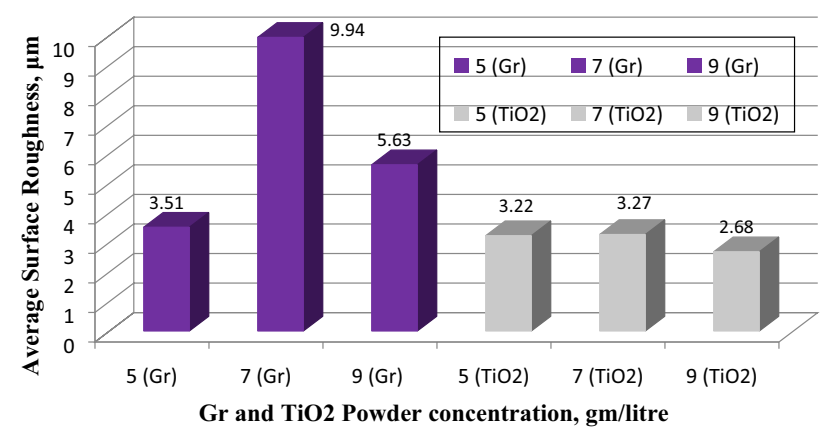

Fig. 9 Bar charts of Average Surface Roughness for different concentrations of $\mathrm{Gr}$ and $\mathrm{TiO} 2$ powders at the peak current value of $20 \mathrm{~A}$
Influence of Powder concentration on MRR ( $\mathrm{mm} 3 / \mathrm{min})$ for $\mathrm{Gr}$ and $\mathrm{TiO} 2$ at peakcurrent of $20 \mathrm{~A}$

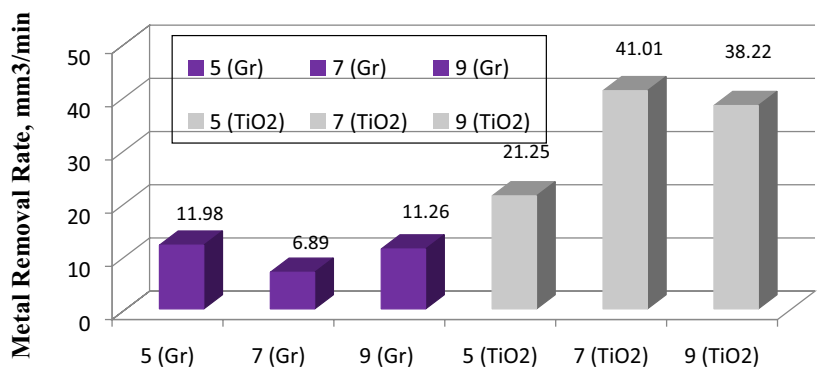

Gr and TiO2 Powder Concentration, g/litre

Fig. 10 Bar charts of Metal Removal Rate for different concentrations of $\mathrm{Gr}$ and $\mathrm{TiO} 2$ powders at the peak current value of $20 \mathrm{~A}$ 
concentration is increased from 7 to $9 \mathrm{gm} / \mathrm{L}$ in the case of Gr powder, though there is a slight fall in the case of $\mathrm{TiO} 2$ powder seen in Fig. 10. A suitable amount of powder leads to the uniform dispersion of discharge energy in every direction, which leads to a decreased surface roughness as the crater size is minimized [34]. Therefore, these findings may be concluded that the successful application of PMEDM, with appropriate selection of the peak current, powder type, and powder concentration leads to higher MRR and lower surface roughness.

Owing to the limited scope of the work, an in-depth study on the HAZ was not included in the current study. The SEM images are presented to have a qualitative assessment of the topography of the HAZ and to explain the surface roughness generated under different machining conditions and powder concentration. SEM images give an indirect idea about the depth of penetration of the effect of the sparking process at the investigated conditions.

\section{Conclusion}

In this study, the effects of concentration (5 -9 g/l) of two powders (Graphite and $\mathrm{TiO}_{2}$ ) and peak current (5A-20A) were investigated on the performance of the PMEDM applied on Ti-6A1-4V (Grade-5) in terms of response parameters-MRR, TWR, and SR. Kerosene was used as the dielectric. The main conclusions drawn on the paper are summarized below:

$\mathrm{TiO}_{2}$ powder showed much superior performance over Graphite powder in terms of all the PMEDM responses; MRR was found to be roughly 3.5 times higher, tool wear ratio (TWR) at the maximum MRR 1.8 times lower, and at the minimum MRR roughly 3 times lower in the case of $\mathrm{TiO}_{2}$ powder. On the other hand, the maximum surface roughness attained with $\mathrm{TiO}_{2}$ powder was $3.265 \mu \mathrm{m}$ against $9.936 \mu \mathrm{m}$ for graphite powder at the highest MRR, and the same attained at the lowest MRR was $2.228 \mu \mathrm{m}$ for TiO2 against $2.411 \mu \mathrm{m}$ for graphite powder. Thus, $\mathrm{TiO} 2$ powder of appropriate concentration and peak current value may be recommended for rough as well as finish PMEDM process of Ti-6Al-4V (Grade-5) alloy as it can generate higher MRR, lower TWR, and relatively lower SR.

Empirical models of MRR, TWR, and SR developed in the work may be used to predict these responses at a $95 \%$ confidence level in terms of the variable parameters of powder concentration and peak current.

One of the limitations of the present work was the limited number of (two) powders, their narrow range of concentration (5-9 g/L), and only one parameter of current (Peak current) considered in the work. Future works may be performed to determine the optimum concentration of powders in a wider range of powder concentrations and optimize other current parameters (Pulse on/off duration) apart from the peak current. Future work also needs to focus on the in-depth study of the HAZ in terms of its depth, composition, and mechanical properties of the zone, as well as the properties of the layer below the HAZ, as that layer plays a vital role in the performance of the part. Secondly, while considering the concentration of the powder, it would perhaps be more appropriate to consider the densities of the powders to have equivalent volume concentration of the powders, not merely their weight percentages, as it is the number of the particles which is important in terms of PMEDM performance. For instance, Graphite Powder with a density of $1.8 \mathrm{~g} / \mathrm{cm}^{3}$ occupies 2.35 times the volume of the same weight of $\mathrm{TiO} 2$ powder with a density of $4.23 \mathrm{~g} / \mathrm{cm}^{3}$. Hence it is expected that the concentration of a lighter powder will have a larger number of ions to assist in the additional arching process during PMEDM.

\section{Declarations}

Conflict of interest The authors declare that they have no conflict of interest.

Open Access This article is licensed under a Creative Commons Attribution 4.0 International License, which permits use, sharing, adaptation, distribution and reproduction in any medium or format, as long as you give appropriate credit to the original author(s) and the source, provide a link to the Creative Commons licence, and indicate if changes were made. The images or other third party material in this article are included in the article's Creative Commons licence, unless indicated otherwise in a credit line to the material. If material is not included in the article's Creative Commons licence and your intended use is not permitted by statutory regulation or exceeds the permitted use, you will need to obtain permission directly from the copyright holder. To view a copy of this licence, visit http://creativecommons .org/licenses/by/4.0/.

\section{References}

1. Saptaji K, Gebremariam MA, Azhari MABM (2018) Machining of biocompatible materials: a review. Int J Adv Manuf Technol 97(5-8):2255-2292. https://doi.org/10.1007/s00170-018-1973-2

2. Bains PS, Bahraminasab M, Sidhu SS, Singh G (2020) On the machinability and properties of Ti-6Al-4V biomaterial with n-HAp powder-mixed ED machining. Proc Inst Mech Eng Part $\mathrm{H}$ J Eng Med 234(2):232-242. https://doi.org/10.1177/0954411919 891887

3. Panda RC, Sharada A, Samanta LD (2021) A review on electrical discharge machining and its characterization. Mater Today Proc. https://doi.org/10.1016/j.matpr.2020.11.546

4. Singh G, Ablyaz TR, Shlykov ES, Muratov KR, Bhui AS, Sidhu SS (2020) Enhancing corrosion and wear resistance of Ti6Al4V alloy 
using CNTs mixed electro-discharge process. Micromachines 11(9):850. https://doi.org/10.3390/MI11090850

5. Ganachari VS et al (2019) A comparative performance study of dry and near dry EDM processes in machining of spring steel material. Mater Today Proc 18:5247-5257. https://doi. org/10.1016/j.matpr.2019.07.525

6. Gouda D, Panda A, Nanda BK, Kumar R, Sahoo AK, Routara BC (2021) Recently evaluated Electrical Discharge Machining (EDM) process performances: a research perspective. Mater Today Proc. https://doi.org/10.1016/j.matpr.2020.12.180

7. Bhui AS, Bains PS, Sidhu SS, G. Singh (2019) Parametric optimization of ED machining of Ti-6Al-4V in CNTs mixed dielectric medium. Mater Today Proc 18:1532-1539. https://doi. org/10.1016/j.matpr.2019.06.623

8. Anwar MM (2008) An experimental investigation on surface characteristics of tool steel using powder mixed micro-EDM.

9. Singh A, Singh R (2015) Effect of powder mixed electric discharge machining (PMEDM) on various materials with different powders: a review. IJIRST-Int J Innov Res Sci Technol 2(03):164-169

10. Jawahar M, Sridhar Reddy C, Srinivas C (2019) A review of performance optimization and current research in PMEDM. Mater Today Proc 19:742-747. https://doi.org/10.1016/j.matpr .2019.08.122

11. Singh G, Sidhu SS, Bains PS, Bhui AS (2019) Improving microhardness and wear resistance of $316 \mathrm{~L}$ by $\mathrm{TiO} 2$ powder mixed electro-discharge treatment. Mater Res Express 6(8):86501. https://doi.org/10.1088/2053-1591/ab1 bab

12. Kumar H, Davim JP (2011) Role of powder in the machining of Al-10\%Sicp metal matrix composites by powder mixed electric discharge machining. J Compos Mater 45(2):133-151. https:// doi.org/10.1177/0021998310371543

13. Cui C, Hu BM, Zhao L, Liu S (2011) Titanium alloy production technology, market prospects and industry development. Mater Des 32(3):1684-1691. https://doi.org/10.1016/j.matde s.2010.09.011

14. Jabbaripour B, Sadeghi MH, Shabgard MR, Faraji H (2013) Investigating surface roughness, material removal rate and corrosion resistance in PMEDM of $\mathrm{Y}$-TiAl intermetallic. J Manuf Process 15(1):56-68. https://doi.org/10.1016/j.jmapro.2012.09.016

15. Pramanik A, Littlefair G (2015) Machining of titanium alloy (Ti-6Al-4V)-theory to application. Mach Sci Technol 19(1):1-49. https://doi.org/10.1080/10910344.2014.991031

16. Hwang YL, Kuo CL, Hwang SF (2010) The coating of TiC layer on the surface of nickel by electric discharge coating (EDC) with a multi-layer electrode. J Mater Process Technol 210(4):642-652. https://doi.org/10.1016/j.jmatprotec.2009.11.013

17. Singh G, Sidhu SS, Bains PS, Singh M, Bhui AS (2020) On surface modification of Ti alloy by electro discharge coating using hydroxyapatite powder mixed dielectric with graphite tool. J Bio- Tribo-Corros 6(3):1-11. https://doi.org/10.1007/s4073 5-020-00389-0

18. Khan AA, Ndaliman MB, Zain ZM, Jamaludin MF, Patthi U (2012) Surface modification using electric discharge machining (EDM) with powder addition. Appl Mech Mater 110-116:725-733.https ://doi.org/10.4028/www.scientific.net/AMM.110-116.725

19. Janmanee $P$, Muttamara A (2012) Surface modification of tungsten carbide by electrical discharge coating (EDC) using a titanium powder suspension. Appl Surf Sci 258(19):7255-7265. https://doi.org/10.1016/j.apsusc.2012.03.054

20. Abdullah RIR, Redzuwan BI, Abdul Aziz MS, Kasim MS (2017) Comparative study of tool wear in milling titanium alloy
(Ti-6AI-4V) using PVD and CVD coated cutting tool. Ind Lubr Tribol 69(3):363-370. https://doi.org/10.1108/ILT-09-2016-0202

21. Liu ZQ, Xu J, Han S, Chen M (2013) A coupling method of response surfaces (CRSM) for cutting parameters optimization in machining titanium alloy under minimum quantity lubrication (MQL) condition. Int J Precis Eng Manuf 14(5):693-702. https:// doi.org/10.1007/s12541-013-0093-z

22. Abdudeen A, Qudeiri JEA, Kareem A, Ahammed T, Ziout A (2020) Recent advances and perceptive insights into powder-mixed dielectric fluid of EDM. Micromachines 11(8):754. https://doi. org/10.3390/MI11080754

23. Kansal HK, Singh S, Kumar P (2007) Technology and research developments in powder mixed electric discharge machining (PMEDM). J Mater Process Technol 184(1-3):32-41. https://doi. org/10.1016/j.jmatprotec.2006.10.046

24. Sidhu SS, Batish A, Kumar S (2014) Study of surface properties in particulate-reinforced metal matrix composites (MMCs) using powder-mixed electrical discharge machining (EDM). Mater Manuf Process 29(1):46-52. https://doi.org/10.1080/10426 914.2013.852211

25. Sahu NK, Andhare AB (2019) Multiobjective optimization for improving machinability of Ti-6Al-4V using RSM and advanced algorithms. J Comput Des Eng 6(1):1-12. https://doi. org/10.1016/j.jcde.2018.04.004

26. Tawfiq MA, Abbas AA, Global journal of engineering science and research management

27. Groover MP (2010) Fundamentals of modern manufacturing: Part 2. Wiley, New Jersey

28. Ho KH, Newman ST (2003) State of the art electrical discharge machining (EDM). Int J Mach Tools Manuf 43(13):1287-1300. https://doi.org/10.1016/S0890-6955(03)00162-7

29. Rahman M, Senthil Kumar A, Prakash JRS (2001) Micro milling of pure copper. J Mater Process Technol 116(1):39-43. https:// doi.org/10.1016/S0924-0136(01)00848-2

30. Anayet M, Patwari U, Amin AKMN, Arif MD (2011) Optimization of surface roughness in end milling of medium carbon steel by coupled statistical approach with genetic algorithm. First Int Conf Interdiscip Res Dev 19:41.1-41.5

31. Luo YF (1997) The dependence of interspace discharge transitivity upon the gap debris in precision electrodischarge machining. J Mater Process Technol 68(2):121-131. https://doi. org/10.1016/S0924-0136(96)00019-2

32. Makwana AV, Banker KS (2015) An experimental investigation on AISI 316 stainless steel for tool profile change in die sinking EDM using DOE. Sch J Eng Technol 3:447-462

33. Al-Khazraji A, Amin SA, Ali SM (2016) The effect of SiC powder mixing electrical discharge machining on white layer thickness, heat flux and fatigue life of AISI D2 die steel. Eng Sci Technol Int J 19(3):1400-1415. https://doi.org/10.1016/j.jestch.2016.01.014

34. Kolli M, Kumar A (2015) Effect of dielectric fluid with surfactant and graphite powder on electrical discharge machining of titanium alloy using taguchi method. Eng Sci Technol Int J 18(4):524-535. https://doi.org/10.1016/j.jestch.2015.03.009

Publisher's Note Springer Nature remains neutral with regard to jurisdictional claims in published maps and institutional affiliations. 